\title{
O ADOLESCENTE E A COMPETÊNCIA SOCIAL: FOCANDO O NÚMERO DE AMIGOS
}

\section{ADOLESCENTAND SOCIAL COMPETENCE: FOCUSING ON THE NUMBER OF FRIENDS}

Maria Marta da Silva $^{1}$
Teresa Helena Sclhoen-Ferreira $^{2}$
Élide Medeiros $^{3}$
Maria Aznar-Farias ${ }^{4}$
${\text { Mércia Regina Marcondes Pedromônico }{ }^{5}}$

SILVA, M.M.D.; SCHOEN-FEREIRA T.H.; MEDEiROS, E.; AZNAR-FARIAS, M.; PEDROMÔNICO, M.R.M. O adolescente e a competência social: focando o número de amigos. Rev. Bras. Cresc. Desenv. Hum., Sao Paulo, 14(1): 28-34, 2004.

Resumo: Na adolescência, a convivência com o grupo de pares é fundamental. A amizade propicia prazer e traz benefícios para o desenvolvimento do indivíduo. Objetivo: verificar o número de amigos íntimos que os adolescentes que procuraram um centro de saúde dizem ter, verificando se o sexo ou a adequação idade-série escolar interferem no número de amigos Método: estudo descritivo de corte transversal. Foram escolhidos aleatoriamente 250 prontuários, dos quais 197 (78,8\%) preencheram os requisites dos estudos, sendo 62,44\% do sexo feminino. Os adolescentes tinham de I I a 18 anos e todos frequentavam Escola de Ensino Fundamental ou Médio. Instrumento: Foi utilizado o Youth Self Report - YSR (ACH EN BACH, 199 1), uma escala de rastreamento de problemas de comportamento e competência social. Procedimento: Os adolescentes que frequentam o Centro de Atendimento e Apoio ao Adolescente - CAAA - foram entrevistados com o YSR no período de 1999 a 2002. Resultados: Mais meninas responderam ter de 2 a 3 amigos íntimos, enquanto mais meninos responderam ter quatro ou mais. Os adolescentes, em geral, se consideram bons amigos e sem dificuldade para ter amizade. Discussão: Meninas parecem ser mais seletivas quanto à amizade do que meninos. Os adolescentes apresentaram uma boa auto-imagem quanto ao relacionamento com amigos. Conclusão: Os adolescentes estão realizando uma das tarefas evolutivas desta fase: ter amigos íntimos.

Palavras-chave: adolescência; competência social; amizade.

\section{INTRODUÇÃO}

Adolescência é o modo de experimentar a puberdade, sofrendo as influências culturais e sociais (AZNAR-FARIAS, no prelo). Dentre estas, podemos citar a amizade.
Como em outros períodos da vida, o adolescente necessita de apoio solidário para enfrentar as situações ligadas a seu crescimento e desenvolvimento. Nesta fase, o grupo assume um papel fundamental e será o lugar onde o adolescente encontrará a oportunidade de aprender a

Trabalho apresentado como parte dos requisitas para obtenção, pela primeira autora, do titulo de especialista em Adolescência para Equipe Multidisciplinar - área Psicologia, coordenado pelas duas últimas autoras.

1 Especialista em Adolescência pelo CAAA- UNIFESP

2 Mestre em Ciências Aplicadas à Pediatria pela UNIFESP

3-4-5 Profs. Adjuntos da UNIFESP

Endereço para correspondência: Universidade Federal de São Paulo/Escola Paulista de Medicina - Centro de Atendimento e Apoio ao Adolescente Rua Botucatu, 7^15 - Tel.: 5576-4360. CEP: 04()23-062 - São Paulo - SP. E-mail: adolescenc.dped@epm.br 
compartilhar sentimentos e enfrentar as várias transformações para o desabrochar da maturidade sem a interferência dos pais.

O grupo de pares pode ser considerado "um laboratório social”, onde as relações igualitárias e recíprocas permitem a exploração de diversas fonnas de ser (SCHOEN-FERREIRA, 2002). O grupo ajuda na transição da vida familiar para a vida independente do mundo adulto. Nele, o jovem tem a oportunidade de experimentar situações novas, dividir responsabilidades e aprender com o erro dos demais. Desta fonna, o adolescente prepara-se para as novas tarefas que assumirá.

As amizades são relações especiais entre duas pessoas que compartilham momentos específicos de suas vidas, onde existem laços de afetividade, reciprocidade e fidelidade (KIMMEL \& WEINER, 1998). Estes relacionamentos são movidos pela proximidade, intimidade, comprometimento e lealdade, onde o ser humano é respeitado e aceito pelo que é (THOMAS \& DALBMAN, 2001). É essencial pertencer a um grupo de iguais e ser aceito pelos seus membros (FAU, 1954; HERBERT, 1991).

O grupo nasce da necessidade de segurança e de participação emocional com outros iguais. São formados pela carência de laços permanentes e íntimos, para preencher um vazio e vivenciar relações afetivas. No grupo de pares, o adolescente tem a sensação de pertinência, pode confrontar-se e reconhecer-se (KIMMEL \& WEINER, 1998). Seu ego passa a ser contemplado no espelho dos outros, onde todos vivenciam as mesmas transformações físicas e emocionais, porém o trabalho de elaboração é individual para que o adolescente alcance sua universalidade (PEDROMÔNICO, 2000).

Ter amigos é inerente à condição humana e vem sendo considerado um indício de adequação social. A amizade propicia prazer e traz benefícios para o desenvolvimento do indivíduo. Os adolescentes beneficiam-se mais dos relacionamentos entre pares que as crianças mais novas (THOMAS \& DAUBMAN, 2001).

A amizade íntima é algo pessoal que envolve sentimentos e processos de comunicação verbal e não-verbal, bem como comportamentos e modos de organização onde as pessoas compartilham momentos específicos de suas vidas (MARQUES, 1993). O estudo de PACHECO (1999) confirmou estes pressupostos, salientando a importância das habilidades sociais nos relacionamentos interpessoais. Estas habilidades são conhecimentos e práticas de comportamentos, para que o indivíduo obtenha sucesso na interação com outros, por exemplo, iniciar e manter uma conversação. Inabilidades de linguagem parecem constituir-se um fator de risco para comportamento anti-social (RUTTER, 2003).

Pessoas inseridas socialmente possuem um suporte emocional e com isso estão menos propensas a sofrer distúrbios emocionais e mentais. Os indivíduos sem amigos apresentam poucas habilidades sociais ou trazem consigo algum transtorno de personalidade (NEWCOMB et al., 1993; KIMMEL \& WEINER, 1998). A confiança, presente na amizade, favorece a expressao de pensamentos e sentimentos, sendo uma via de mão dupla, permitindo ao amigo a oportunidade de compreender os pensamentos e sentimentos do outro.

A maioria das pessoas têm muitos conhecidos que se tratam amistosamente com quem passam grande parte do seu tempo. Porém, o verdadeiro amigo é aquele que é capaz de sacrificar-se pelo outro amigo, de preocupar-se com o seu bemestar, de auxiliá-lo nas dificuldades, de compartilhar seus segredos e evitar magoá-lo (KIMMEL \& WEINER, 1998). Para saber a intensidade da amizade e o quanto ela é íntima, basta avaliar o quanto a pessoa revela de si para uma outra e o quanto ela sabe sobre as características pessoais e íntimas do amigo. As qualidades de um verdadeiro amigo citadas por adolescentes são semelhantes às citadas em estágios posteriores.

Os autores divergem na quantidade de pessoas para compor um grupo de iguais. KIMMEL e WEINER (1998) consideram as panelinhas como pequenos grupos sociais onde os participantes estão estreitamente ligados, variando de 5 a 10 integrantes. DUNPHY (1963) designou o grupo constituído por 4 a 6 jovens com altos níveis de intimidade de panelinha. Para JAEHN et al. (1988), estes grupos recebem o nome de turma.

No decorrer da adolescência, o relacionamento do jovem com amigos e conhecidos atinge novos níveis de intensidade e multiplica-se em número (HERBERT, 1991; BEE, 1996; KIMMEL \& WEINER, 1998). Porém é pouco provável que o adolescente num grupo maior tenha desejo de expor suas dificuldades e fragilidade. $\mathrm{O}$ amigo íntimo continua sendo importante.

Os adolescentes possuem expectativas diferentes sobre amizade de acordo com o sexo (THOMAS \& DAUBMAN, 2001). O adolescente do sexo masculino possui, com seus amigos próximos, uma relação interpessoal e não individual. E as adolescentes do sexo feminino valorizam mais os relacionamentos sociais. As amizades entre as meninas são mais íntimas e com auto-revelação. No entanto, independente do sexo, os adolescentes consideram a amizade importante para sua vida (SCHOENFERREIRA, et al., 2003).

WALDROP e HALVENSON (1975) consideraram os relacionamentos masculinos como 
extensivos e os femininos como intensivos, visto os grupos de meninos terem mais membros e estes se reunirem para atividades físicas, sendo menos propícios a trocas confidenciais, embora pareça não haver diferença no número de amigos íntimos entre os sexos.

Em um estudo brasileiro sobre as características para a escolha dos amigos, as meninas deram maior ênfase do que os meninos na importância do amigo ser educado e simpático (21\%), enquanto 8\% dos meninos citaram estas características (JAEHN et al., 1988).

Alguns pesquisadores salientaram a dificuldade de encontrar dados sobre a amizade na adolescência, especialmente relações de intimidade do adolescente com sua família nuclear e com seus melhores amigos (FIELD et al., 1995).

CLAES (1998) estudou a interferência cultural no tempo despendido pelo adolescente no relacionamento com os pais e com grupo de amigos. Seus resultados mostram que os canadenses compartilham mais tempo com os amigos do que com os pais; os italianos participam mais das atividades em família do que com os amigos; e os belgas ocupam tempo relativamente igual com os pais e seus parceiros de idade. Concluiu, então, que o desenvolvimento das relações interpessoais na adolescência depende do contexto cultural.

$\mathrm{Na}$ nossa sociedade, o adolescente deve freqüentar a escola. Esta o mantém implicado em experiências de aprendizagem que desenvolvem não só destrezas cognitivas, mas também aperfeiçoam as habilidades sociais (SCHOEN-FERREIRA, no prelo).

A escola representa para o adolescente o grupo social mais amplo. Através das relações com os pares terá a oportunidade de experimentar momentos marcantes em sua vida, além da possibilidade de vivenciar momentos de afetividade, podendo se reconhecer e se constituir enquanto pessoa e fazer escolhas com o apoio do grupo. A escola é uma instituição formadora, porque propicia vivências individuais para o crescimento global e trocas interpessoais significativas (CÁRDENAS, 2002).

Ao pesquisarem a inserção de adolescentes brasileiros de 15 a 18 anos em grupo de iguais, JAEHN et al. (1988) observaram que a metade dos jovens tinha muitos amigos e muitos conhecidos, sendo que $85 \%$ pertenciam à "turma" e $42,5 \%$ disseram pertencer a um grupo, embora não participassem de todas as atividades da turma ou grupo. Afirmaram não pertencer a nenhuma turma, $12,5 \%$.

PILON (1984) destacou que, dentre os tópicos da rotina de vida dos adolescentes, ter colegas, companheiros e amigos representa o item mais importante. Para os adolescentes de ambos os sexos a importância do grupo de pares teve uma das pontuações mais altas, para o sexo masculino 80,5\% e para o sexo feminino $86,3 \%$.

HUSSONG (2000), em sua pesquisa, encontrou que as adolescentes do sexo feminino variavam de 1 a 20 amigos próximos e os do sexo masculino de 0 a 15. Ambos os sexos descreveram a amizade como algo bonito. Os adolescentes relataram que estas amizades não são recentes, as meninas j á desfrutam destas amizades por um período de 3 a 5 anos, enquanto os meninos, de 4 a 5 anos.

Um estudo transversal, da adolescência à meia idade, observou que a quantidade de amigos aumenta com a idade, porém na adolescência os amigos se encontram muito mais, passam mais tempo juntos que em qualquer outra fase do desenvolvimento humano (PAPALIA \& OLDS, 1985).

Apesar de os jovens terem menos amigos que os adultos, dão muito maior importância a eles. Com isto, podem ter expectativas pouco realistas da quantidade de amigos que deveriam ter, e ficarem frustrados com o número que têm (PAPALIA \& OLDS, 1985).

Considerando como premissa que adolescentes inseridos em grupos aprimorem suas competências sociais e se envolvam em interações com outros jovens e que estas interações acrescem e modificam habilidades cognitivas e sociais, este estudo teve por objetivo verificar o número de amigos íntimos que os adolescentes que procuraram um centro de saúde dizem ter, verificando se o sexo ou a adequação idade-série escolar interferem no número de amigos.

\section{MATERIAL E MÉTODO}

Este estudo descritivo de corte transversal envolveu a análise de questionários aplicados aos adolescentes atendidos de 1999 a 2002 no Centro de Atendimento e Apoio ao Adolescente - CAAA - do Departamento de Pediatria da Universidade Federal de São Paulo Unifesp. Tal centro atende todos os adolescentes que procuram o ambulatório de Pediatria para o seguimento de rotina pediátrica ou por queixas fisicas (crescimento, obesidade, etc.) ou de comportamento social e/ ou de habilidades acadêmicas. O CAAA conta com profissionais de saúde de diferentes especialidades, visando atenção integral ao desenvolvimento saudável do adolescente.

\section{Participantes}

Participaram deste estudo 250 adolescentes que responderam ao 7 YSR. Foram ex- 
cluídos aqueles que não estavam cursando o ensino básico, como universitários ou deficientes mentais, e os que não se encontravam na faixa de aplicação do instrumento (11 a 18 anos), participando do estudo 197 adolescentes, sendo a maioria dos adolescentes do sexo feminino.

\section{Instraumento}

Neste trabalho foi utilizado o Young SelfReport - YSR -, que é a forma dirigida ao adolescente de 11 a 18 anos do Child Behavior Checklist (ACHENBACH, 1991), para triagem de problemas de comportamento e competência social. Por seu rigor metodológico de construção, é utilizado em diferentes culturas e mostra sensibilidade e especificidade para a finalidade a que se destina em todas elas.

Dentre as questões de competência social, duas foram selecionadas para o nosso estudo, aquela em que o adolescente assinala quantos amigos íntimos possui: nenhum; um; dois ou três; quatro ou mais; e a que ele indica como percebe o seu relacionamento com outros adolescentes.

\section{Procedimento}

Na sala de espera para a consulta, foi feito o primeiro contato com o adolescente e o convite para responder o YSR em forma de entrevista. Para este estudo, escolheu-se aleatoriamente 250 questionários, dos quais foram retiradas as respostas dadas aos dois itens escolhidos, além do sexo, idade e escolaridade do adolescente. Com essas informações, construiu-se um banco de dados (programa Excel). Os protocolos foram separados por sexo e por faixa etária, buscando com isto estudar a interferência destes fatores no número de amigos, ainda levando em conta a adequação e defasagem escolar para o estudo. As três faixas etárias foram estabelecidas a partir do critério descrito pela literatura. Os adolescentes entrevistados estavam esperando o atendimento nutricional, gran- de parte por obesidade; o atendimento psicológico, grande parte por comportamento antisocial; o atendimento psicopedagógico, por mau desempenho escolar; ou o atendimento médico, para triagem ou atendimento de seguimento, na sua maioria.

Para determinar o nível de defasagem escolar, compreendeu-se a diferença de dois anos ou mais em relação à série escolar prevista para sua idade cronológica. Todos os participantes deste estudo estavam inseridos no meio acadêmico, estudando em escolas de ensino básico (Ensino Fundamental e ou Ensino Médio).

\section{RESULTADOS}

A Figura l apresenta a distribuição do número de amigos referidos pelos adolescentes do CAAA.

Percebe-se, através dos dados, que prevaleceu entre os adolescentes do sexo masculino a resposta quatro ou mais amigos íntimos, e entre os do sexo feminino a opção dois a três amigos. Nesta amostra não houve diferença do número de amigos entre faixa etária.

Todos os adolescentes da amostra estavam cursando o Ensino Fundamental ou Ensino Médio. A maioria dos adolescentes estava adequada em relação à idade cronológica e série escolar $(83,76 \%)$ (Tabela 1).

Não houve diferença entre estar defasado na escola e o número de amigos assinalados. Na faixa etária dos 17 e 18 anos, observa-se a maior defasagem independente do sexo (Figura 2).

Estes dados evidenciam que a maioria dos adolescentes que freqüentam o CAAA percebemse tendo um bom relacionamento com outros jovens, igual a qualquer outro adolescente, pois lhe é perguntado a sua percepção de relacionamento em comparação com outros jovens da mesma idade. Não foi estudado se existe uma relação entre o motivo de procura ao CAAA e o número de amigos.

Tabela 1. Distribuição do número de amigos referidos dos adolescentes com defasagem escolar e sexo segundo a faixa etária

\begin{tabular}{ccccccc}
\hline & $\begin{array}{c}\mathbf{1 1} \\
\text { Feminino }\end{array}$ & $\begin{array}{c}\mathbf{1 3} \text { anos } \\
\text { Masculino }\end{array}$ & $\begin{array}{c}\mathbf{1 4} \\
\text { Feminino }\end{array}$ & $\begin{array}{c}\mathbf{1 6} \text { anos } \\
\text { Masculino }\end{array}$ & $\begin{array}{c}\mathbf{1 7} \\
\text { Feminino }\end{array}$ & $\begin{array}{c}\mathbf{1 8} \text { anos } \\
\text { Masculino }\end{array}$ \\
\hline 0 & $0(0 \%)$ & $1(50 \%)$ & $2(33,33 \%)$ & $2(22,22 \%)$ & $0(0 \%)$ & $0(0 \%)$ \\
1 & $0(0 \%)$ & $1(50 \%)$ & $2(33,33 \%)$ & $2(22,22 \%)$ & $0(0 \%)$ & $0(0 \%)$ \\
2 ou 3 & $2(66,66 \%)$ & $0(0 \%)$ & $2(33,33 \%)$ & $2(33,33 \%)$ & $5(50 \%)$ & $1(50 \%)$ \\
4 ou 7 & $3(33,33 \%)$ & $0(0 \%)$ & $0(0 \%)$ & $3(33,33 \%)$ & $2(20 \%)$ & $0(0 \%)$ \\
\hline Total & $\mathbf{3}$ & $\mathbf{2}$ & $\mathbf{6}$ & $\mathbf{9}$ & $\mathbf{1 0}$ & $\mathbf{2}$ \\
\hline
\end{tabular}




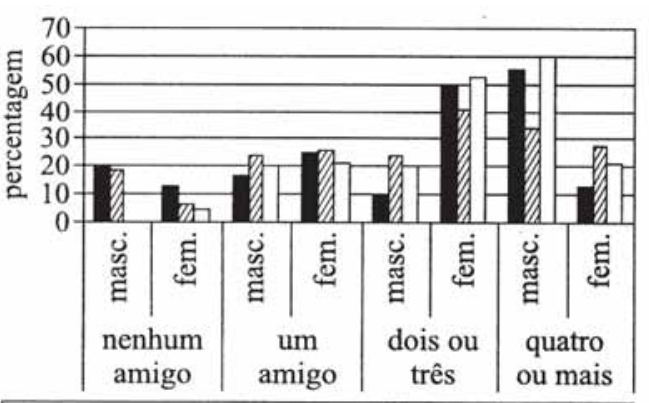

11 a 13 anos $\square 14$ a 16 anos $\square 17$ a 18 anos

Figura 1. Distribuição do número de amigos referidos segundo o sexo e faixa etária, em porcentagem.

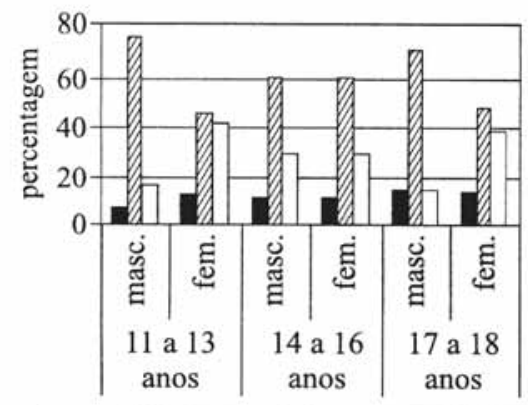

pior $\square$ igualmente bem $\square$ melhor

Figura 2. Distribuição dos adolescentes segundo sua percepção de relacionamento com outros adolescentes, em porcentagem.

\section{DISCUSSÃO}

Em relação ao número de amigos, poucos adolescentes responderam assinalando a opçao “0” (zero) amigos. Para os meninos houve a tendência de responder terem de 4 ou mais amigos íntimos; para as meninas, dois ou três amigos. A literatura mostra que as meninas são mais seletivas quanto às suas amizades, por isso é de se esperar que digam ter menos amigos que os meninos. Ao mesmo tempo em que vemos ser absolutamente comum o adolescente de ambos os sexos afirmarem possuir muitos amigos (HUSSONG, 2000). O adolescente responder que possui dois, três ou quatro amigos, sugere que possui habilidades sociais para manter uma amizade sólida, e, a partir disto, podemos inferir que tem competência social.

Manter uma amizade significa desenvolver as habilidades de confiar no outro e ser uma pessoa confiável, procurar não ofender o amigo, de recorrer ao outro em busca de apoio emocio- nal. Para manter este relacionamento é preciso aprender a compartilhar mais e competir menos e desenvolver a lealdade. Os adolescentes costumam defender seus amigos. Estas são também características de amizade para os adultos, o que vai indicando a transição da adolescência para o próximo estágio do ciclo vital.

Para manter um relacionamento mais íntimo e duradouro com alguém, é preciso ser capaz de expressar seus próprios pensamentos e sentimentos, mas também considerar o ponto de vista do outro. Desenvolve-se, assim, a habilidade inata do ser humano de ser leitor de mentes (TREVARTHEN \& AITKEN, 1994; BARONCOHEN, 1995), de perceber a intencionalidade dos atos, não se atendo aos mesmos. O entendimento que o adolescente tem dos outros repete o seu desenvolvimento cognitivo (BEE, 1996), ou seja, a sua capacidade de compreender a perspectiva de outra pessoa.

Os dados parecem mostrar que os adolescentes que estão defasados em relação à série escolar dizem ter menos amigos que aqueles com adequação escolar. A literatura nos informa uma grande relação entre sucesso acadêmico e inserção nos grupos de pares (KIMMEL \& WEINER, 1998). Os adolescentes rejeitados tendem a se reunir em grupos, não por escolha de amizade, mas por falta desta opção (DIAS, 2001). A própria defasagem escolar pode ser devida ao seu desenvolvimento cognitivo e, paralelamente, a cognição social não vai sendo desenvolvida no mesmo ritmo. Além do mais, estar atrasado implica em cursar uma série com adolescentes bem mais novos, onde os interesses são muito diferentes. Isto dificulta ainda mais criar e manter a amizade. $\mathrm{O}$ entendimento cognitivo dos relacionamentos sociais não vem antes do próprio relacionamento. É vivenciando os relacionamentos que se desenvolve a cognição social, e desenvolvendo este aspecto, pode-se ampliar e aprofundar os relacionamentos sociais.

A Lei de Diretrizes e Bases da Educação Nacional (Lei $n^{\circ}$ 9394/96) organizou o processo educativo em ciclos, tendo como eixo o desenvolvimento humano, colocando não só a transmissão de conhecimentos, mas também a possibilidade de vivências sociais e culturais, tentando transformar a concepção de educação ao atingir também a rede social que envolve os alunos. Ao prever a progressão regular por série, onde, dentro de cada ciclo educacional não há repelência, permite que o aluno se encontre com outros da mesma idade e provavelmente com os mesmos interesses.

Neste estudo, quando analisamos a percepção que os adolescentes disseram ter de seus rela- 
cionamentos com outros jovens, observamos que a maioria dos meninos se viu dando-se tão bem com outros adolescentes quanto estes entre si. Já as meninas, se viram igual ao grpo de pares, ou relacionando-se melhor que os outros adolescentes da mesma faixa etária. Perceber-se igual aos outros ajuda no desenvolvimento da auto-imagem.

Uma das grandes questões da adolescência é considerá-la um “aborrecente”, aquele que atrapalha o convívio social dos outros. Ao perceber-se como igual aos outros, o adolescente está dizendo que possui qualidades singulares e comportamentos característicos. Os adolescentes entrevistados apresentaram uma boa auto-imagem quanto ao aspecto relacionamento com grupo de iguais. Os grupos estigmatizados desenvolvem sua auto-estima valorizando aspectos específicos nos quais se destacam e fazendo o que todos os outros do grupo fazem (MYERS, 1999). Os adolescentes entrevistados valorizaram a amizade, procurando ter contato com os pares, tendo atividades em conjunto, como conversar, fazer trabalhos escolares, ir a shoppings, jogar futebol, sair da escola em grupo.

Os adolescentes do sexo masculino que freqüentam o CAAA disseram ter quatro ou mais amigos íntimos, e as adolescentes dois ou três. Não houve diferenças em relação ao número de amigos e a defasagem escolar ou idade.

Ao observarmos que os adolescentes dizem ter amigos (para meninas, dois ou três; para meninos, quatro ou mais) e que se dão tão bem com eles quanto outros adolescentes, observamos que são congruentes, estão consistentes em suas experiências, suas idéias, percepções e valores sobre amizade.

A amizade é fundamental para um desenvolvimento saudável e de manutenção da vida social. Querer e ter amigos faz parte da condição humana. É importante que mais pesquisas sejam realizadas dando ênfase ao relacionamento de intimidade dos adolescentes com seus pares, na busca de conhecimento para o desenvolvimento da competência social.

Finalizando, é importante que outras pesquisas sejam realizadas dando ênfase ao relacionamento de intimidade dos adolescentes e seus pares. Nosso estudo limitou-se a levantar o número de amigos referidos pelo adolescente, sem caracterizar o grau de intimidade, ou o que cada adolescente entende por amizade. Nesta linha devem ser endereçados novos estudos. Além disto, observa-se a necessidade de modelar a relação sexo, número de amigos e escolaridade do adolescente, utilizando modelo de regressão.

\begin{abstract}
In adolescence, living in a same-aged group is fundamental. Friendship provides pleasure and brings benefits to the individual's development. Objective: to ascertain the number of close friends that adolescents who attended a health centre say they have, checking if sex or the con-ectness of the school level/age relation affect the number of friends. Method: transversal descriptive research. 250 individuais were chosen at random, 197 of them (78.8\%) fulfilled the characteristics of this study, with $62.44 \%$ of female presence. The adolescents were between 11 and 18 years old and ali studied at primary or secondary schools. Instrument: we utilized the "Youth Self Report" - YSR (Achenbach, 1991), a system for tracking behavioural and social competence problems. Procedure: The adolescents attending the "Centro de Atendimento e Apoio ao Adolescente - CA^AA” (Centre for Adolescent Assistance and Support) were interviewed with the YSR from 1999 until 2002. Results: most of lèmales answered they have 2-3 close friends, while most of males, 4 or more. The adolescents, in general, thinkofthemselves asbeing goodfriends andhavenodifficultyinmaking friends. Discussion: females seem to be more selective concerning friendship compared to males. The adolescents showed a good self-image regarding the relationship with friends. Conclusion: the adolescents are ca^ $\wedge \sim$ ying out one of the evolutionary tasks of this phase: to have close friends.
\end{abstract}

Key-words: adolescence; social competence; friendship.

\section{REFERÊNCIAS BIBLIOGRÁFICAS}

Achenbach TM. Manual for the Youth Self Report and 1991 profile. Burlington, University of Vermont,1991.

Aznar-farias M. Adolescência normal: quando diagnosticar? In Secretaria Estadual de Saúde SP. Adolescência e Saúde 111. São Paulo, Secretaria Estadual de Saúde, (no prelo).
Baron-Cohen. Mindblindness - An essay on autism and Theory of Mind. Cambridge, Bradford Books, 1995.

Bee H. A criança em desenvolvimento. $7^{\mathrm{a}}$ ed. Porto Alegre, Artes Médicas, 1996.

Cárdenas CJ. Eu, a procura de mim... em/me encontro nos outros. In Sociedade Brasileira de Psicologia. In: Reunião Anual de Psicologia, 
32, Florianópolis, 2002. Resumos de Comunicação Científica, 2002. p. 59-60.

Claes M. Adolescent's closeness with parents, siblings, and friends in three countries: Canada, Belgium and Italy. Journal of Youth and Adolescence 27(2): 165-184, 1998.

Dias MDF. (2001). Adolescentes infratores e nãoinfratores: uma análise comparativa através do CBCL e YSR. São Paulo, 2001. [Dissertação de Mestrado - Universidade Federal de São Paulo]. http://www.brazilpednews.org.br/pos/ mestrado/ tese01/delfina.htm

Dunphy DC. The social structure of urban adolescent peer groups. Sociometry, 26, 230246, 1963.

Fau, R. Los grupos de niños y de adolescentes. Barcelona, Paideia, 1954. p. 55-56.

Griffin K (2003). Amigos: o segredo para uma vida mais longa. Seleções Reder’s Digest, janeiro, 60-65, 2003.

Field T, Lang C, Yando R, Bendele D. Adolescents'intimacy with parents and friends. Adolescence, 30(117): 132-140, 1995.

Herbert M. Convivendo com adolescentes. Rio de Janeiro, Bertrand-Brasil, 1991.

Hussong AM. Distinguishing mean and structural sex differences in adolescent friendship quality. Journal of Social and Personal Relationships, 17(2): 223-243, 2000.

Jaehn SM, Goldberg TBL, Kfouri JRN, Simões AXP, Curi PR. Avaliação do desenvolvimento afetivo social do adolescente na faixa etária dos 15 aos 18 anos: Estudo com adolescentes do municipio de Botucatu - São Paulo. J. Pediatr, 64(4), 111-114, 1988.

Jersild AT. Psicologia da adolescência. Rio de Janeiro, Nacional, 1969.

Kimmel DC, Weiner IB. La adolescencia: una transición del desarrollo. Barcelona, Editorial Ariel, 1998.

Marques JC. Relações interpessoais e processos de grupo: O conceito de intimidade. Psicologia, 2(24): 9-22, 1993.

Myers D. Introdução à Psicologia Geral. $5^{\mathrm{a}}$ ed. Rio de Janeiro, LTC,1999. p. 309.

Newcomb AF, Buowisky WM, Pattee L. Children's peer relations: a meta-analytic review of popular, rejected, neglected, controversial and average sociometric status. Psychology, 113: 99-128, 1993.
Pacheco JTB. Estilos parentais e desenvolvimento de habilidades sociais na adolescência. Psicologia: Teoria e Pesquisa, 2: 117- 126, 1999.

Papalia DE, Olds SW. Desarrollo Humano. México, McGraw-Hill, 1985.

Papalia DE, Olds SW. Desenvolvimento Humano. $7^{\mathrm{a}}$ ed. Porto Alegre, Artes Médicas, 2000.

Pedromônico MRM. A sexualidade do adolescente In: Terapêutica e Prática Pediátrica. $2^{a}$ ed. São Pau1o, Atheneu, 2000, p. 472-475.

Pilon AF. Desenvolvimento na adolescência: Sexualidade, interação com os pais, companheiros e sexo oposto. São Paulo, 1984. [Tese de doutorado Universidade de São Paulo].

Rutter M. Commentary: causal processes leading to antisocial behavior. Developmental Psychology, 39(2): 372-378, 2003.

Schoen-Ferreira TH, Aznar-Farias M, Silvares EFM. A construção da identidade em adolescentes: um estudo exploratório. Estudos de Psicologia, 8(1): 107-116, 2003.

Schoen-Ferreira TH. O adolescente e a escola. In Secretaria Estadual de Saúde SP. Adolescência e Saúde III. São Paulo, Secretaria Estadual de Saúde, (no prelo).

Schoen-Ferreira TH, Alves D, Aznar-Farias M, Silvares EFM. Perfil e principais queixas dos clientes encaminhados ao Centro de Atendimento e Apoio Psicológico ao Adolescente (CAAA) Unifesp/EPM. Psicologia em estudo, 7(2): 7382, 2001.

Thomas J, Daubman KA. The relations between friendships: quality and self-esteem in adolescent girl and boys. Sex Roles, 45: 53-64, 2001.

Trevarthen C, Aitken KJ. Brain Development, Infant Communication, and Empathy Disorders: Intrinsic Factors in Child Mental Health. Development Psychopatology, 6:597633, 1994.

Waldrop MF, Halvenson CF. Intensive and extensive peer behavior: Longitudinal and crosssectional analysis. Child Development, 46: 1926, 1975. 